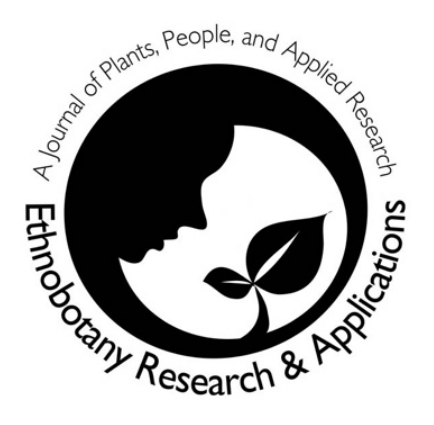

\section{An overview of Ethnomedicinal plants of Uzbekistan}

\author{
Olim K. Khojimatov, Dilovar T. Khamraeva, Alisher N. \\ Khujanov and Rainer W. Bussmann
}

\section{Databases and Inventories}

\begin{abstract}
Background: The Flora of Central Asia is extremely rich, with about 9800 species of vascular plants. Uzbekistan, with over 4500 species, has a central position in the region, and is regarded as one of the main centers of medicinal plants diversity, in particular because of its high percentage of local endemism. About 600 species of medicinal plants have been documented in Uzbekistan for the treatment of numerous diseases, many of them endemic. These plants are still used for the medicinal traits in industrial scale as well as by local tabibs (local practitioner).
\end{abstract}

Methods: During research, semi-structured interviews were conducted with tabibs, elders and herders with experience in healing by using medicinal plants. During expedition visits, herbarium material and samples of medicines from vegetative raw materials were collected. Collected materials were determined using local floristic monographs and herbarium vouchers.

Results: During fieldwork, we recorded 117 medicinal plants belonging to 45 families and 94 genera, used widely for the treatment of various diseases. Traditional practitioners prepared the plants mostly as extract, decoction, powder, bathing and sometimes used them fresh. Extract and decoction were generally preferred, and the preparations were normally made fresh just before use. Some of the encountered plants are now also used by private entrepreneurs for the production of herbal medicines for various purposes.

Conclusions: Our results show that despite the achievements of modern allopathic medicine, the population in Uzbekistan continues to actively use wild medicinal plants for health purposes. The wide availability of medicinal plants, low costs, and perceived efficacy contribute to popularization of traditional medicine.
Keywords: Ethnobotany; ethnopharmacology; medicinal plants; Uzbekistan; Central Asia.

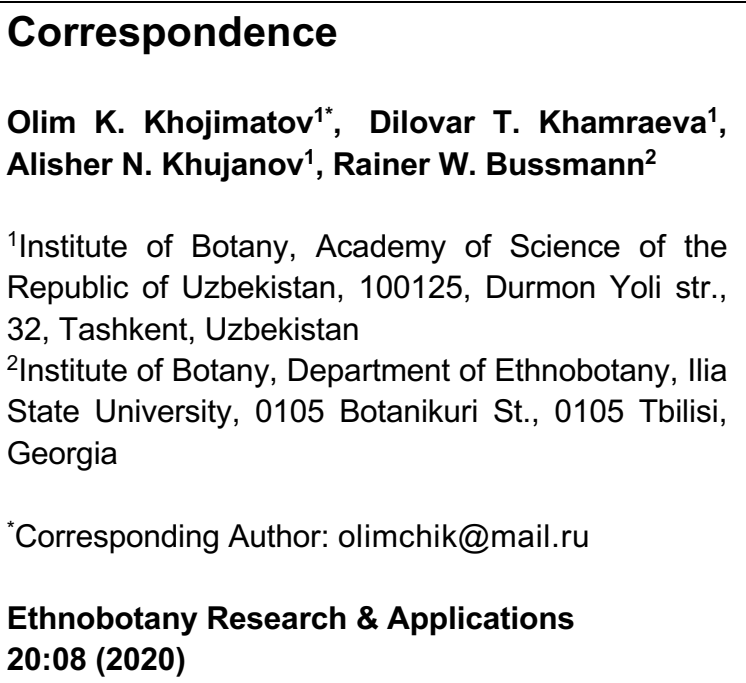

\section{Xulosa}

Ma'lumot: O'rta Osiyo florasi juda boy bo'lib, 9800 ga yaqin yursak o'simliklari mavjud (Xasanov 2015). 4500 dan ortiq turga ega bo'lgan O'zbekiston mintaqada markaziy mavqega ega va dorivor o'simliklar xilma-xilligining asosiy markazlaridan biri hisoblanadi, qisman uning mahalliy endemizm darajasi juda yuqori. O'zbekistonda ko'plab kasalliklarni davolash uchun dorivor o'simliklarning 600 ga yaqin turi hujjatlashtirilgan, ularning ko'plari endemikdir. Ushbu o'simliklar hanuzgacha sanoat miqyosida dorivor xususiyatlari uchun mahalliy amaliyotchilar tomonidan qo'llaniladi.

Usullari: Tadqiqot davomida dorivor o'simliklardan foydalangan holda davolash tajribasiga ega bo'igan tabiblar, oqsoqollar va cho'ponlar bilan intervyu o'tkazildi. Ekspeditsiya safarlari davomida gerbariy materiallari va o'simlik xom ashyosidan olingan dorilar namunalari to'plangan. To'plangan materiallar mahalliy floristik monografiyalar, o'simliklar 
aniqlagichlari va gerbariy namunalari yordamida aniqlandi.

Natijalar: Ushbu maqolada ko'p yillik etnobotanik tadqiqotlar natijalari tahlil qilingan bo'lib, unda O'zbekistonda an'anaviy tibbiyotda yovvoyi o'simliklarning 100 dan ortiq turlariqo'llanilishi hamda ulardan hozirda xususiy tadbirkorlar tomonidan turli maqsadlarda ishlatiladigan dorivor preparatlar ishlab chiqarishda foydalanilishi keltirilgan.

Xulosa: Bizning natijalarimiz zamonaviy ilmiy tibbiyotda erishilgan yutuqlarga qaramay, O'zbekiston aholisi yovvoyi dorivor o'simliklardan davolanish uchun faol foydalanishda davom etishini ko'rsatdi. Bunda dorivor o'simliklar ta'sir darajasini keng qamrovligi, tabiiyligi va arzonbopligi an'anaviy tibbiyotni ommalashtirishga yordam beradi.

Kalit so'zlar: Etnobotanika; etnofarmakologiya; dorivor o'simliklar; O'zbekiston; O'rta Osiyo.

\section{Background}

There are some regions in the world that have been very important for the development of human civilization. Central Asia - the region between the Caspian Sea and Siberian Altay Mountains, features prominently among them. According to Nicolai Vavilov (Vavilov 1992), Central Asia is one of the eight centers of origin and diversity of cultivated plants. Many domestic species originated from this region, including apples, dwarf wheat, lentils, and garlic. Many wild relatives of onion, apple, spinach, and almond, to name a few, still grow there along with other wild relative species of crops. The region was and is still botanically extremely rich and has a lot of promising economic and ornamental plants that have not yet been explored or introduced into international horticulture. Central Asia includes five countries of the former Soviet Union: Kazakhstan, Kyrgyzstan, Tajikistan, Turkmenistan, and Uzbekistan.

The long isolation of these countries, both from one another and from the world, has prevented them from undertaking a coordinated, regional approach toward understanding and conserving their interrelated floras. Central Asia extends $1300 \mathrm{~km}$ from north to south and $2400 \mathrm{~km}$ from west to east. The large Turanian Lowland forms the northern part of the region. It gradually rises in the southeastern part to high mountain borders. Large areas are occupied by deserts and by high mountains, including the Tien Shan and Pamir-Alay which harbor numerous plateaus that range from 3700 to over $5000 \mathrm{~m}$. Fittingly, Pamir means "roof of the World" in the Tajik language. The Flora of Central Asia is extremely rich, with about 9800 species of vascular plants (Khassanov 2015). Uzbekistan, with over 4500 species, has a central position in the region. The southern border of the region runs along Iran, Afghanistan and China. About 600 plant species are used in traditional medicine, but only around 200 species have been phytochemically investigated, and some 150 plants species were included in the original Pharmacopoeia of Uzbekistan (Khojimatov et al. 1993). Traditional medicine in Central Asia has a long history, which began many centuries ago, but its most notable period was in the $10^{\text {th }}-11^{\text {th }}$ centuries. Many early scientists tried to explore the secrets of folk medicines, including, Abu Raihon Beruni (973 1048) (Figure 1) and Abu Ali ibn Sino (Avicenna) (980-1037) (Figure 2), both of which contributed greatly to the early codification of herbal medicines.

Avicenna was born in the territory of modern Uzbekistan, near Bukhara. He wrote 456 books, among them 62 books about medicines. The most famous part of his work is the "Canon" in 5 volumes (Ibn Sina 1982, Uzbek edition), the second volume of which was dedicated to medicinal plants, describing about 1500 drugs and almost 800 species of medicinal vascular plants. Historically Uzbek and Tajik botanists, especially in $20^{\text {th }}$ century made significant contributions to develop pharmacognosy, pharmacology and phytotherapy (Khojimatov \& Kobetc 1988; Khojimatov \& Olloyorov 1988; Kholmatov et al. 1984).

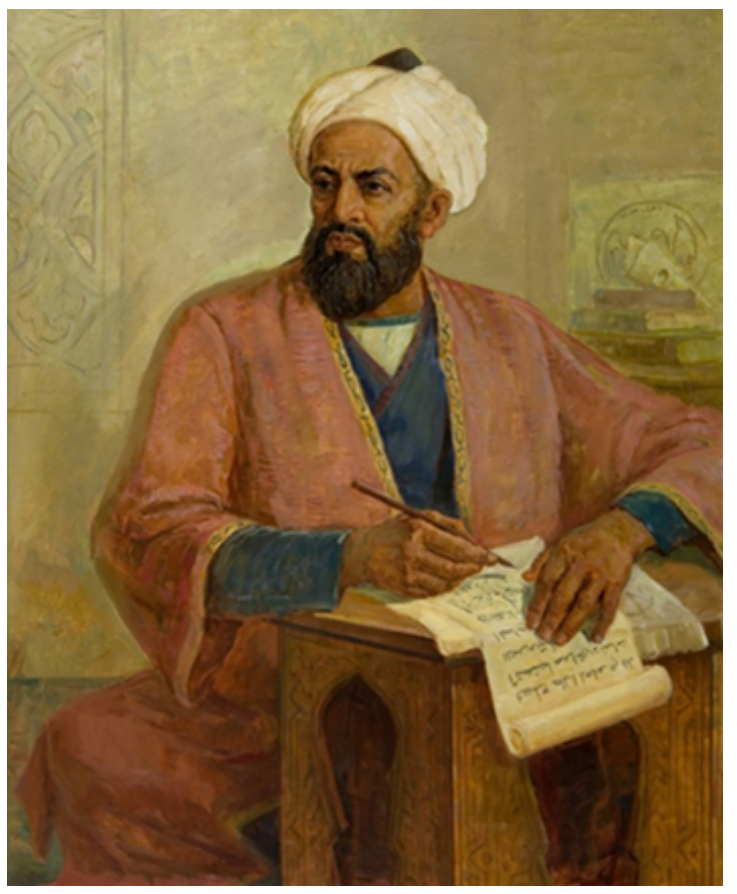

Figure 1. Abu Raykhon Beruny 


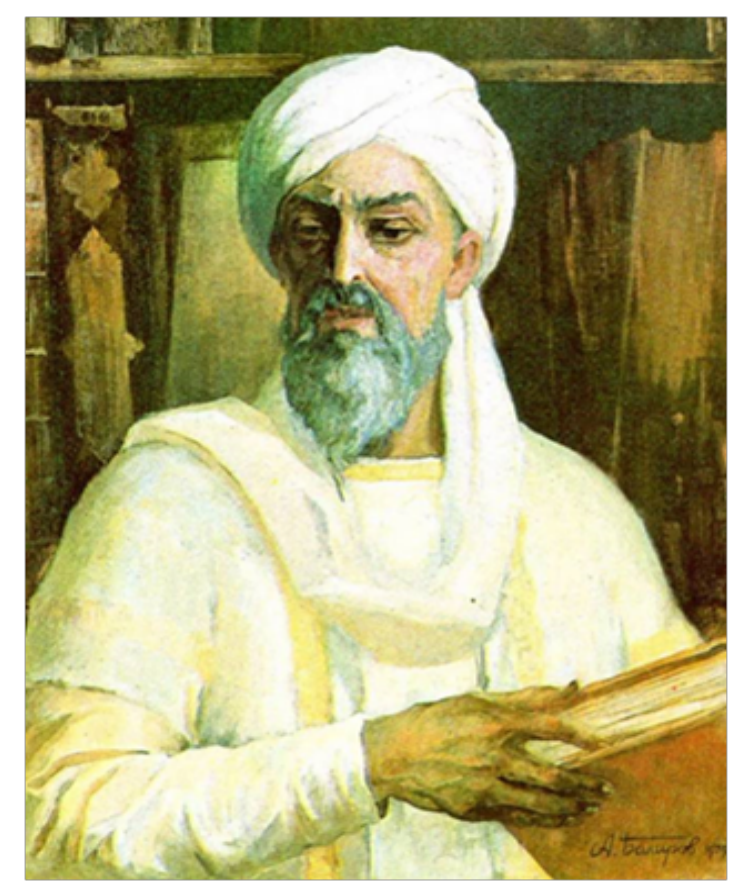

Figure 2. Avicenna

Since Uzbekistan's independence, the use of medicinal herbs in the treatment of various diseases has been significantly developed. This was facilitated by measures taken by the State in terms of popularizing the centuries-old experience of folk medicine, through the creation of the Academy of Folk Medicine of Uzbekistan, the Ibn Sina International Foundation (Avicenna), as well as the way out of the shadow of folk healers (tabibs), which in previous times was not welcome treatment by folk means. All this led to the further popularization of the traditional medicine and the local population began to use herbs more confidently. Often, herbal preparations have a wider spectrum of action compared to synthetic preparations, and, in view of their naturalness, are often more tolerable to the human body, due to less toxicity. Nowadays pharmacy chains and markets are selling medicinal plants in natureal state, as well as, extracts and tinctures, especially of popular species like Ziziphora pedicellata, Origanum tyttanthum, Matricaria chamomilla, Achillea millefolium, among others.

The purpose of this work was to identify wild medicinal plants of the flora of Uzbekistan used by the local population for medicinal purposes, and to analyze and systematize the information received.

\section{Materials and Methods}

Classical ethnobotanical methods were used for gathering information obtained from the mountain villages of Uzbekistan and provided by the local tabibs, schoolteachers and shepherds. Participants were selected by snowball sampling and gave their prior informed consent before conducting semistructured interviews using a standard questionnaire. Plant samples were collected on walks in the fields. Ethnobotanical studies were carried out in the spring - autumn periods of 2014-2019. The Kashkadarya, Samarkand and Tashkent regions of the territory of the Republic of Uzbekistan were examined. Information was collected in mountain settlements by interviewing tabibs, elders, shepherds and other storage media, of which 9 interviewees were tabibs, 9 were elders and 7 shepherds. As a rule, representatives of the male part of the population are engaged in traditional medicine in Central Asia, as a result of which, all the respondents were men. For ethnobotanical work, we used a specially developed questionnaire to elucidate more detailed information convenient for further analysis (Khojimatov, 2008). The age of the interviewees ranged from 38 to 84 years. Interviews were conducted in Uzbek, Tadjik and Russian after receiving prior informed consent. It must also be emphasized that many tabibs are however not willing to disclose their knowledge about treatment methods and the plants used, since traditionally the experience of the healer is transmitted only to family members from generation to generation and, therefore, the recipes for preparing treatment are kept secret.

All specimens have been taxonomically analyzed, and all herbarium samples are stored in the Herbarium (TASH) of the Institute of the Botany Academy of Sciences, Uzbekistan. The nomenclature of species was revised in using www.tropicos.org, and family classification follows APGIII (APG 2009).

\section{Results}

During fieldwork, we recorded 117 medicinal plants used widely for the treatment of various diseases (Table 1). Traditional practitioners prepared the plants mostly as extract, decoction, powder, bathing and sometimes used them fresh. Extract and decoction were generally preferred, and the preparations were normally made fresh just before use. For some plants like Issirik (Peganum harmala), the aerial part was burned, and the smoke was inhaled, for treatment and prophylactics of infectious diseases, such as influenza, as also reported by other studies (Astulla et al. 2008; Moloudizargari et al. 2013). Species had often multiple uses, and e.g. 24 medicinal species were commonly used as food (Table 1). These plants were also used for the preventive treatment of diseases such as vitamin deficiency and common cold. 
Table 1. The most using species in traditional medicine of Middle Asia

\begin{tabular}{|c|c|c|c|c|c|c|}
\hline Scientific name & Local name & Part used & $\begin{array}{c}\text { Forms of } \\
\text { preparation }\end{array}$ & Traditional uses & Remarks & $\begin{array}{l}\text { Herbarium samples voucher } \\
\text { (TASH) }\end{array}$ \\
\hline \multicolumn{7}{|l|}{ Amaryllidaceae } \\
\hline Allium karataviense Regel & Piyoz & bulbs & fresh & common cold & $E, F$ & $\begin{array}{l}\text { specimen voucher K. Tojibaev, } \\
\text { 07.06.2015. №7 }\end{array}$ \\
\hline Allium suworowii Regel & Piyoz & bulbs & fresh & $\begin{array}{l}\text { skin diseases, vitamin } \\
\text { deficiency }\end{array}$ & $\mathrm{F}$ & $\begin{array}{l}\text { specimen voucher } \mathrm{N} \text {. } \\
\text { Beshko,12.05.2012.№258 }\end{array}$ \\
\hline Ungernia sewerzowii (Regel) B. Fedtsch. & Qoraqovuk & $\begin{array}{l}\text { bulbs, } \\
\text { leaves }\end{array}$ & extract & $\begin{array}{l}\text { bronchitis, for treatment } \\
\text { of wounds }\end{array}$ & $E$ & $\begin{array}{l}\text { specimen voucher G. Khamidov, } \\
\text { 24.05.1987. №37 }\end{array}$ \\
\hline Ungernia nvictoris Vved. ex Artjush. & Omonqora & $\begin{array}{l}\text { bulbs, } \\
\text { leaves }\end{array}$ & extract & poliomyelitis & $E$ & $\begin{array}{l}\text { specimen voucher K. Khozhimatov, } \\
\text { 09.07.1960. №232 }\end{array}$ \\
\hline \multicolumn{7}{|l|}{ Anacardiaceae } \\
\hline Pistacia vera $\mathrm{L}$. & Pista & galls & extract & $\begin{array}{l}\text { cardiac and respiratory } \\
\text { diseases }\end{array}$ & $\mathrm{F}$ & $\begin{array}{l}\text { specimen voucher A. Kazakbaev, } \\
\text { 09.06.1986. №136 }\end{array}$ \\
\hline Rhus coriaria L. & Tatum & $\begin{array}{l}\text { leaves, } \\
\text { fruits }\end{array}$ & decoction & $\begin{array}{l}\text { hypertension, gastric } \\
\text { ulcer }\end{array}$ & & $\begin{array}{l}\text { specimen voucher M. Nabiev, } \\
\text { 03.06.1972. №405 }\end{array}$ \\
\hline \multicolumn{7}{|l|}{ Apiaceae } \\
\hline $\begin{array}{l}\text { Elwendia persica (Boiss.) Pimenov \& } \\
\text { Kljuykov }\end{array}$ & Zira & seeds & decoction & $\begin{array}{l}\text { stomach, cardiac } \\
\text { diseases }\end{array}$ & $\mathrm{F}$ & $\begin{array}{l}\text { specimen voucher G. Gaffarov, } \\
\text { 27.07.1986. №5335 }\end{array}$ \\
\hline Ferula foetida Regel & $\begin{array}{l}\text { Sassiq } \\
\text { kovrak }\end{array}$ & gum & fresh, extract & $\begin{array}{l}\text { for wounds, diabetes, } \\
\text { tuberculosis }\end{array}$ & & $\begin{array}{l}\text { specimen voucher N. Beshko, } \\
\text { 05.05.2011. №005 }\end{array}$ \\
\hline Heracleum lehmannianum Bunge & Boldirgan & $\begin{array}{l}\text { leaves, } \\
\text { roots }\end{array}$ & extract & $\begin{array}{l}\text { dental and skin } \\
\text { diseases, convulsion }\end{array}$ & & $\begin{array}{l}\text { specimen voucher M. Nabiev, } \\
\text { 31.05.1980. № } 502\end{array}$ \\
\hline $\begin{array}{l}\text { Mediasia macrophylla (Regel et } \\
\text { Schmalh.) Pimenov }\end{array}$ & Alqoruti & a. p. & fresh, extract & $\begin{array}{l}\text { liver and gall-bladder } \\
\text { diseases, rheumatism, } \\
\text { nephritis, eczema } \\
\text { (Kurimoto et al., 2011; } \\
\text { Khojimatov et al., 2015) }\end{array}$ & $E, F$ & $\begin{array}{l}\text { specimen voucher A. Pyatayeva, } \\
\text { 14.07.1976.№2 }\end{array}$ \\
\hline Prangos pabularia Lindl. & Shashir & a. p. & extract & $\begin{array}{l}\text { external for skin } \\
\text { diseases }\end{array}$ & & $\begin{array}{l}\text { specimen voucher M. Nabiev, } \\
\text { 31.05.1980. №515 }\end{array}$ \\
\hline \multicolumn{7}{|l|}{ Apocynaceae } \\
\hline \multirow[t]{2}{*}{ Vinca erecta Regel \& Schmalh. } & Barigul & $\begin{array}{l}\text { whole, } \\
\text { plants }\end{array}$ & decoction & $\begin{array}{l}\text { headache, cardiac } \\
\text { diseases }\end{array}$ & \multirow[t]{2}{*}{$\mathrm{E}, \mathrm{T}$} & \multirow[t]{2}{*}{$\begin{array}{l}\text { specimen voucher E. Korotkova, } \\
\text { 26.06.1967. №1163. }\end{array}$} \\
\hline & & & powder & external for wounds & & \\
\hline Araceae & & & & & & \\
\hline
\end{tabular}




\begin{tabular}{|c|c|c|c|c|c|c|}
\hline \multirow[t]{2}{*}{ Acorus calamus $\mathrm{L}$. } & \multirow[t]{2}{*}{ Igir } & rhizome & powder & liver cancer & & \multirow{2}{*}{$\begin{array}{l}\text { specimen voucher N. Naralieva, } \\
\text { 30.05.2015.№56 }\end{array}$} \\
\hline & & & extract & $\begin{array}{l}\text { diuretic, tonic for } \\
\text { central nervous system }\end{array}$ & & \\
\hline \multicolumn{7}{|l|}{ Asteraceae } \\
\hline Achillea filipendulina Lam. & Dastarbosh & a. p. & extract & stomach diseases & & $\begin{array}{l}\text { specimen voucher A. Yuldashev, } \\
\text { 01.06.1987. №12 }\end{array}$ \\
\hline Achillea millefolium L. & Buymadaron & a.p. & extract & stomach diseases & & $\begin{array}{l}\text { specimen voucher A. Egamberidev, } \\
\text { 14.06.2001.№64 }\end{array}$ \\
\hline Arctium tomentosum Mill. & Qarikiz & $\begin{array}{l}\text { leaves, } \\
\text { roots }\end{array}$ & decoction & $\begin{array}{l}\text { rheumatism, external } \\
\text { skin diseases }\end{array}$ & & $\begin{array}{l}\text { specimen voucher Yu. Grigoriev, } \\
\text { 06.07.1959. № } 32 \text {. }\end{array}$ \\
\hline Artemisia absinthium L. & Erman & a.p. & extract & $\begin{array}{l}\text { liver, kidney, } \\
\text { sleeplessness }\end{array}$ & & $\begin{array}{l}\text { specimen voucher K. Djabbarov, } \\
\text { 23.07.1983. №530 }\end{array}$ \\
\hline Artemisia dracunculus L. & Sherolgin & a.p. & extract & stomach diseases & & $\begin{array}{l}\text { specimen voucher K. Toyjanov, } \\
\text { 14.07.1996. №101 }\end{array}$ \\
\hline Bidens tripartita L. & Ititkanak & leaves & bath & skin diseases & & $\begin{array}{l}\text { specimen voucher S. } \\
\text { Sokhobiddinov, 09.06.1949. №1849 }\end{array}$ \\
\hline Centaurea cyanus L. & Butakuz & flowers & decoction & fever & & $\begin{array}{l}\text { specimen voucher O. Turginov, } \\
\text { 10.07.2012. №87 }\end{array}$ \\
\hline Centaurea. depressa M. Bieb. & Bulakuz & flowers & decoction & common cold & & $\begin{array}{l}\text { specimen voucher T. Makhkamov, } \\
\text { 25.04.2006.№26 }\end{array}$ \\
\hline Cichorium intybus L. & Sachratki & roots & decoction & cardiac diseases & & $\begin{array}{l}\text { specimen voucher T. Makhkamov, } \\
\text { 01.05.2005. №8. }\end{array}$ \\
\hline $\begin{array}{l}\text { Helichrysum maracandicum Popov ex } \\
\text { Kirp. }\end{array}$ & Ulmasut & flowers & decoction & $\begin{array}{l}\text { liver and gall-bladder } \\
\text { diseases }\end{array}$ & $E$ & $\begin{array}{l}\text { specimen voucher A. Khujanov, } \\
\text { 03.07.2018. №38 }\end{array}$ \\
\hline Inula macrophylla Kar. \& Kir. & Sariqandiz & roots & extract & $\begin{array}{l}\text { intestinal diseases, } \\
\text { laxative }\end{array}$ & & $\begin{array}{l}\text { specimen voucher M. Nabiev, } \\
\text { 23.07.1988. №174 }\end{array}$ \\
\hline Inula helenium L. & Qoraandiz & roots & extract & $\begin{array}{l}\text { blood coagulant, } \\
\text { laxative }\end{array}$ & & $\begin{array}{l}\text { specimen voucher M. Nabiev, } \\
\text { 18.06.1980. №108 }\end{array}$ \\
\hline Onopordum acanthium L. & Oqqarak & a.p. & decoction & $\begin{array}{l}\text { astma, cought, high } \\
\text { blood pressure }\end{array}$ & & $\begin{array}{l}\text { specimen voucher E. Kortokova, } \\
\text { 25.06.1978. №229 }\end{array}$ \\
\hline Rhaponticum integrifolium C. Winkl. & Levzeya & roots & extract & as a tonic, diabetes & & $\begin{array}{l}\text { specimen voucher I. } \\
\text { Raikova,01.09.1987.№1535 }\end{array}$ \\
\hline Tanacetum pseudachillea C. Winkl. & Dastarbosh & a.p. & decoction & laxative & & $\begin{array}{l}\text { specimen voucher I. } \\
\text { Khamidhodjaev, 25.07.1973.№78 }\end{array}$ \\
\hline $\begin{array}{l}\text { Taraxacum officinale (L.) Weber ex F.H. } \\
\text { Wigg. }\end{array}$ & Koki & $\begin{array}{l}\text { leaves, } \\
\text { roots }\end{array}$ & extract & $\begin{array}{l}\text { diuretic, laxative, liver } \\
\text { diseases }\end{array}$ & & $\begin{array}{l}\text { specimen voucher S. Kovalevskaya, } \\
\text { 14.04.1961. №45 }\end{array}$ \\
\hline
\end{tabular}




\begin{tabular}{|c|c|c|c|c|c|c|}
\hline Tussilago farfara L. & Oqqaldirmoq & a.p. & decoction & asthma, cough & & $\begin{array}{l}\text { specimen voucher O. Turginov, } \\
\text { 23.04.2012. }\end{array}$ \\
\hline Xanthium strumarium L. & Guzatkon & $\begin{array}{l}\text { fruits, } \\
\text { leaves }\end{array}$ & extract & dysentery, wounds & & $\begin{array}{l}\text { specimen voucher R. } \\
\text { Yesemuradova, 16.05.2010. } \\
\text { №0214. }\end{array}$ \\
\hline \multicolumn{7}{|l|}{ Berberidaceae } \\
\hline Berberis integerrima Bunge & Zirk & bark & decoction & $\begin{array}{l}\text { liver and kidnes } \\
\text { chseascs }\end{array}$ & $\mathrm{F}$ & $\begin{array}{l}\text { specimen voucher M. Nabiev, } \\
\text { 25.05.1978. №758 }\end{array}$ \\
\hline Berberis oblonga (Regel) C.K. Schneid. & Qorazirak & fruits & extract & $\begin{array}{l}\text { rheumatism, bruise and } \\
\text { wounds }\end{array}$ & $\mathrm{F}$ & $\begin{array}{l}\text { specimen voucher } \mathrm{H} \text {. } \\
\text { Mirzakarimova, } 16.06 .2008 \text {. №201 }\end{array}$ \\
\hline \multicolumn{7}{|l|}{ Brassicaceae } \\
\hline Capsella bursa-pastoris (L.) Medik. & Jagjag & a. p. & decoction & blood coagulant & & $\begin{array}{l}\text { specimen voucher E. Sulaymonov, } \\
\text { 23.05.2006. №2 }\end{array}$ \\
\hline \multicolumn{7}{|l|}{ Campanulaceae } \\
\hline $\begin{array}{l}\text { Codonopsis clematidea (Schrenk ex } \\
\text { Fisch. \& C.A. Mey.) C.B. Clarke }\end{array}$ & Dugboy & a. p. & decoction & liver, nausea, skin rash & $E$ & $\begin{array}{l}\text { specimen voucher K. Tojibaev, } \\
\text { 11.06.2004. №56 }\end{array}$ \\
\hline \multicolumn{7}{|l|}{ Capparaceae } \\
\hline \multirow[t]{2}{*}{ Capparis spinosa L. } & Kovar & Roots & extract & $\begin{array}{l}\text { rheumatism, liver } \\
\text { diseases }\end{array}$ & & \multirow[t]{2}{*}{$\begin{array}{l}\text { specimen voucher L. Botirova, } \\
01.10 .2010 \text {. №32 }\end{array}$} \\
\hline & & fruits & decoction & jaundice, diabetes & & \\
\hline \multicolumn{7}{|l|}{ Caryophyllaceae } \\
\hline $\begin{array}{l}\text { Allochrusa gypsophiloides (Regel) } \\
\text { Schischk. }\end{array}$ & Yetmak & roots & extract & bronchitis, cough & & $\begin{array}{l}\text { specimen voucher K. Toyjanov, } \\
\text { 24.07.1996. №118 }\end{array}$ \\
\hline \multicolumn{7}{|l|}{ Chenopodiaceae } \\
\hline Anabasis aphylla L. & Itsigak & shoots & extract & $\begin{array}{l}\text { lung tuberculosis, } \\
\text { asthma }\end{array}$ & $\mathrm{T}$ & $\begin{array}{l}\text { specimen voucher K. Buriev, } \\
\text { 17.10.1974.№95 }\end{array}$ \\
\hline Salsola richteri (Moq.) Kar. ex Litw. & Cherkez & a.p. & extract & $\begin{array}{l}\text { hypertension, } \\
\text { headache (Pak et al., } \\
\text { 2019) }\end{array}$ & & $\begin{array}{l}\text { specimen voucher U. Pratov, } \\
\text { 18.05.1976. №584 }\end{array}$ \\
\hline \multicolumn{7}{|l|}{ Convolvulaceae } \\
\hline Convolvulus arvensis L. & Quypechak & $\begin{array}{l}\text { leaves, } \\
\text { roots }\end{array}$ & extract & laxative, wounds & & $\begin{array}{l}\text { specimen voucher E. Abdullajanov, } \\
\text { 2.7.1974. №214 }\end{array}$ \\
\hline $\begin{array}{l}\text { Convolvulus subhirsutus Regel \& } \\
\text { Schmalh. }\end{array}$ & Ming bosh & a.p. & decoction & convulsion, asthma & $\mathrm{T}$ & $\begin{array}{l}\text { specimen voucher N. Beshko, } \\
\text { 16.07.2012. №264 }\end{array}$ \\
\hline
\end{tabular}




\begin{tabular}{|c|c|c|c|c|c|c|}
\hline Crassulaceae & & & & & & \\
\hline $\begin{array}{l}\text { Rhodiola heterodonta (Hook. f. \& Thoms.) } \\
\text { Boriss. }\end{array}$ & Oltin ildiz & roots & ethanol & tonic, impotence & $\mathrm{T}$ & $\begin{array}{l}\text { specimen voucher I. Levichev, } \\
\text { 17.06.1980. №90 }\end{array}$ \\
\hline \multicolumn{7}{|l|}{ Cupressaceae } \\
\hline $\begin{array}{l}\text { Juniperus polycarpos var. seravschanica } \\
\text { (Kom.) Kitam. }\end{array}$ & Archa & fruits & extract & $\begin{array}{l}\text { kidney, liver, urinary } \\
\text { bladder diseases }\end{array}$ & & $\begin{array}{l}\text { specimen voucher V. Titov, } \\
\text { 10.08.1932.№132. }\end{array}$ \\
\hline $\begin{array}{l}\text { Junioperus pseudosabina Fisch. \& } \\
\text { C.A.Mey. }=\text { J. turkestanica Kom. }\end{array}$ & Urik archa & fruits & extract & rheumatism & & $\begin{array}{l}\text { specimen voucher V. Titov, } \\
\text { 10.08.1932.№127. }\end{array}$ \\
\hline \multicolumn{7}{|l|}{ Elaeagnaceae } \\
\hline Elaeagnus angustifolia L. & Jiyda & $\begin{array}{l}\text { leaves, } \\
\text { fruits }\end{array}$ & extract & bruise and wounds, & $\mathrm{F}$ & $\begin{array}{l}\text { specimen voucher H. Khaydarov, } \\
\text { 12.06.2018. №56 }\end{array}$ \\
\hline Hippophae rhamnoides L. & Chakanda & fruits & oii & $\begin{array}{l}\text { uterine cervical erosion, } \\
\text { for burn }\end{array}$ & $\mathrm{F}$ & $\begin{array}{l}\text { specimen voucher H. Khaydarov, } \\
\text { 12.06.2018. №12 }\end{array}$ \\
\hline \multicolumn{7}{|l|}{ Ephedraceae } \\
\hline Ephedra equisetina Bunge & Togqizilcha & shoots & extract & $\begin{array}{l}\text { common cold, gastric } \\
\text { ulcer, diabetes }\end{array}$ & & $\begin{array}{l}\text { specimen voucher K. Borlev, } \\
\text { 28.05.1981. №377 }\end{array}$ \\
\hline Ephedra intermedia Schrenk \& C.A. Mey. & Qizilcha & shoots & extract & $\begin{array}{l}\text { bronchial asthma, } \\
\text { rheumatism }\end{array}$ & & $\begin{array}{l}\text { specimen voucher U. Pratov, } \\
\text { 14.05.1975. №54 }\end{array}$ \\
\hline \multicolumn{7}{|l|}{ Euphorbiaceae } \\
\hline Euphorbia jaxartica Prokh. & Sutlama & latex & fresh & wart and corn & $\mathrm{E}, \mathrm{T}$ & $\begin{array}{l}\text { specimen voucher I. Maltsev, } \\
\text { 18.07.1987. №sn }\end{array}$ \\
\hline \multicolumn{7}{|l|}{ Fabaceae } \\
\hline Alhagi pseudalhagi Desv. & Yantoq & a.p. & decoction & fever, diuretic, cough & & $\begin{array}{l}\text { specimen voucher T. Makhkamov, } \\
\text { 15.07.2008. №241 }\end{array}$ \\
\hline Astragalus eximius Bunge & Aslragal & a.p. & extract & cough & & $\begin{array}{l}\text { specimen voucher M. Nabiev, } \\
\text { 25.05.1980. № } 145\end{array}$ \\
\hline Astragalus sieversianus Pall. & Pahtak & a.p. & extract & $\begin{array}{l}\text { epilepsy, nervous } \\
\text { system diseases }\end{array}$ & & $\begin{array}{l}\text { specimen voucher U. Pratov, } \\
\text { 22.05.1976 №636 }\end{array}$ \\
\hline Cullen drupaceum (Bunge) C.H. Stirt. & Oqqurai & $\begin{array}{l}\text { leaves, } \\
\text { roots }\end{array}$ & powder & eczema, prolapsed & & $\begin{array}{l}\text { specimen voucher A.Kuziev, } \\
\text { 15.06.2006. №52 }\end{array}$ \\
\hline Glycyrrhiza glabra L. & Shirinmiya & roots & decoction & $\begin{array}{l}\text { cardiac and respiratory } \\
\text { diseases }\end{array}$ & & $\begin{array}{l}\text { specimen voucher O. Turginov, } \\
\text { 13.06.2011. №0062 }\end{array}$ \\
\hline Melilotus officinalis (L.) Lam. & $\begin{array}{l}\text { Qashqar } \\
\text { beda }\end{array}$ & a.p. & extract & laxative, cough & & $\begin{array}{l}\text { specimen voucher T. } \\
\text { Makhkamov,06.07.2006.№312 }\end{array}$ \\
\hline
\end{tabular}




\begin{tabular}{|c|c|c|c|c|c|c|}
\hline Thermopsis alterniflora Regel \& Schmalh & Afsonak & a.p. & extract & bronchitis, pneumonia & $\mathrm{T}$ & $\begin{array}{l}\text { specimen voucher R. } \\
\text { Halmukhamedova, 19.06.1975. } \\
\text { №69 }\end{array}$ \\
\hline Thermopsis lanceolata $\mathrm{R} . \mathrm{Br}$. & Afsonak & a.p. & extract & bronchitis, pneumonia & $\mathrm{T}$ & $\begin{array}{l}\text { specimen voucher K. Toyjanov, } \\
\text { 17.09.1978. №5269. }\end{array}$ \\
\hline \multicolumn{7}{|l|}{ Grossulariaceae } \\
\hline Ribes nigrum L. & Qorakat & fruits & $\begin{array}{c}\text { fresh, } \\
\text { decoction }\end{array}$ & $\begin{array}{l}\text { gastric ulcer, common } \\
\text { cold }\end{array}$ & $\mathrm{F}$ & $\begin{array}{l}\text { specimen voucher O. Turginov, } \\
\text { 29.09.2006.№231 }\end{array}$ \\
\hline \multicolumn{7}{|l|}{ Hypericaceae } \\
\hline Hypericum elongatum C.A. Mey. & Dalachoi & a.p. & decoction & $\begin{array}{l}\text { blood coagulant, } \\
\text { antiseptic }\end{array}$ & & $\begin{array}{l}\text { specimen voucher H. Esankulov, } \\
\text { 26.06.2010. №265 }\end{array}$ \\
\hline Hypericum scabrum L. & Choiut & a.p. & decoction & $\begin{array}{l}\text { cough, bruise and } \\
\text { wounds }\end{array}$ & & $\begin{array}{l}\text { specimen voucher } A \text {. } \\
\text { Egamberdiev, 11.06.2001.№153 }\end{array}$ \\
\hline Hypericum perforatum $\mathrm{L}$. & Qizil-poicha & a.p. & decoction & $\begin{array}{l}\text { stomach and gall- } \\
\text { bladder diseases }\end{array}$ & & $\begin{array}{l}\text { specimen voucher A. } \\
\text { Egamberdiev, 11.06.2001.№203 }\end{array}$ \\
\hline \multicolumn{7}{|l|}{ Juglandaceae } \\
\hline Juglans regia L. & Yongoq & leaves & extract & $\begin{array}{l}\text { diabetes, skin } \\
\text { tuberculosis }\end{array}$ & $\mathrm{F}$ & $\begin{array}{l}\text { specimen voucher M. Tulaganova, } \\
\text { 10.08.1972. }\end{array}$ \\
\hline Leontice ewersmannii Bunge & Ersovun & tubers & extract & laxative, diuretic & & $\begin{array}{l}\text { specimen voucher R. } \\
\text { Yesemuratova,12.05.2009.№0169. }\end{array}$ \\
\hline Thalictrum foetidum L. & Sanchikut & a.p. & extract & hypertension & & $\begin{array}{l}\text { specimen voucher A. Pyatayeva, } \\
\text { 07.1966. №2091 }\end{array}$ \\
\hline Thalictrum isopyroides C.A. Mey. & Sanchikut & roots & extract & $\begin{array}{l}\text { malaria, jaundice, } \\
\text { tuberculosis }\end{array}$ & & $\begin{array}{l}\text { specimen voucher E. Korovin, } \\
07.1939 \text {. №544. }\end{array}$ \\
\hline \multicolumn{7}{|l|}{ Lamiaceae } \\
\hline Dracocephalum komarovii Lipsky & Buzbosh & a. p. & extract & $\begin{array}{l}\text { high blood pressure } \\
\text { (Uchiyama et al., 2006) }\end{array}$ & $\mathrm{E}$ & $\begin{array}{l}\text { specimen voucher T. } \\
\text { Khudaiberdiev, 30.07.1967.№234 }\end{array}$ \\
\hline Hyssopus seravschanicus (Dub.) Pazij & Kukut & a. p. & extract & $\begin{array}{l}\text { cough, bronchitis, } \\
\text { asthma }\end{array}$ & $\mathrm{E}$ & $\begin{array}{l}\text { specimen voucher K. Djumaev, } \\
\text { 25.07.1972. №23 }\end{array}$ \\
\hline Lagochilus inebrians Bunge & Bozulbang & $\begin{array}{l}\text { flowers, } \\
\text { leaves }\end{array}$ & decoction & $\begin{array}{l}\text { blood coagulant, } \\
\text { hypertension }\end{array}$ & $E$ & $\begin{array}{l}\text { specimen voucher N. Beshko, } \\
\text { 22.07.2007. № } 7068\end{array}$ \\
\hline $\begin{array}{l}\text { Leonurus turkestanicus V.I. Krecz. \& } \\
\text { Kupr. }\end{array}$ & Buyrakchoi & $\begin{array}{l}\text { whole } \\
\text { plants }\end{array}$ & ethanol & $\begin{array}{l}\text { neuro - cardiac } \\
\text { diseases, diabetes }\end{array}$ & & $\begin{array}{l}\text { specimen voucher N. Beshko, } \\
\text { 20.07.2009. № } 9032\end{array}$ \\
\hline Melissa officinalis L. & Limon-ut & a.p. & extract & anemia, asthma & & $\begin{array}{l}\text { specimen voucher G. Khamidov, } \\
\text { 25.06.1973. №37 }\end{array}$ \\
\hline Mentha asiatica Boriss. & Yalpiz & a. p. & decoction & against nausea, cough & & $\begin{array}{l}\text { specimen voucher U. Pratov, } \\
\text { 24.06.1996. №512 }\end{array}$ \\
\hline
\end{tabular}




\begin{tabular}{|c|c|c|c|c|c|c|}
\hline Origanum tyttanthum Gontsch. & Tograihon & a.p. & decoction & laxative, diuretic & $E$ & $\begin{array}{l}\text { specimen voucher G. Goipova, } \\
\text { 04.07.1961. № } 340 \text {. }\end{array}$ \\
\hline Salvia sclarea L. & Mavrak & flowers & extract & oro-dental diseases & & $\begin{array}{l}\text { specimen voucher I. Makhmedov, } \\
\text { 26.06.1978.№41 }\end{array}$ \\
\hline Stachys betoniciflora Rupr. & Togqudusi & a. p. & extract & $\begin{array}{l}\text { menstrual disorders, } \\
\text { neurosis }\end{array}$ & $\mathrm{E}$ & $\begin{array}{l}\text { specimen voucher R. } \\
\text { Kholmukhamedova, 20.06.1975. } \\
\text { №32. }\end{array}$ \\
\hline Ziziphora pedicellata Pazij \& Vved. & Qiyiq-ut & a.p. & extract & $\begin{array}{l}\text { for gall-bladder stones, } \\
\text { hypertension }\end{array}$ & $\mathrm{E}$ & $\begin{array}{l}\text { specimen voucher L. Botirova, } \\
\text { 01.05.2006. №164 }\end{array}$ \\
\hline \multicolumn{7}{|l|}{ Liliaceae } \\
\hline Asparagus officinalis L. & $\begin{array}{l}\text { Dorivor } \\
\text { sarsabil }\end{array}$ & roots & extract & $\begin{array}{l}\text { hydropsy, inflammation } \\
\text { of urinary, bladder }\end{array}$ & $\mathrm{F}$ & $\begin{array}{l}\text { specimen voucher N. Beshko, } \\
\text { 18.07.2015. .№118 }\end{array}$ \\
\hline Asparagus persicus Baker & Sarsabil & roots & extract & hypertension & & $\begin{array}{l}\text { specimen voucher M. Sovetkina, } \\
\text { 1.08.1945. №500 }\end{array}$ \\
\hline \multicolumn{7}{|l|}{ Malvaceae } \\
\hline Althaea armeniaca Ten. & Gulhairi & roots & decoction & respiratory diseases & & $\begin{array}{l}\text { specimen voucher K. Toyjanov, } \\
\text { 22.08.1960.№148 }\end{array}$ \\
\hline Althea officinalis $\mathrm{L}$. & $\begin{array}{l}\text { Dorivor } \\
\text { gulhairi }\end{array}$ & roots & decoction & $\begin{array}{l}\text { respiratory and } \\
\text { stomach diseases }\end{array}$ & & $\begin{array}{l}\text { specimen voucher M. Abdullaev, } \\
\text { 28.07.1968.№2 }\end{array}$ \\
\hline \multicolumn{7}{|l|}{ Moraceae } \\
\hline Ficus carica L. & Anjir & $\begin{array}{l}\text { leaves, } \\
\text { fruits }\end{array}$ & fresh, extract & $\begin{array}{l}\text { skin diseases, common } \\
\text { cold }\end{array}$ & $\mathrm{F}$ & $\begin{array}{l}\text { specimen voucher O. Khassanov, } \\
\text { 30.07.1959. №56 }\end{array}$ \\
\hline \multirow[t]{2}{*}{ Morus alba L. } & Tut & $\begin{array}{l}\text { leaves, } \\
\text { bark }\end{array}$ & decoction & diuretic, hypertension & \multirow[t]{2}{*}{$\mathrm{F}$} & \multirow[t]{2}{*}{$\begin{array}{l}\text { specimen voucher A. Butkov, } \\
\text { 15.05.1955. №69 }\end{array}$} \\
\hline & & fruits & fresh & stomach disorders & & \\
\hline \multirow[t]{2}{*}{ Morus nigra L. } & Qoratut & $\begin{array}{l}\text { leaves, } \\
\text { bark }\end{array}$ & decoction & hypertension & \multirow[t]{2}{*}{$\mathrm{F}$} & \multirow[t]{2}{*}{$\begin{array}{l}\text { specimen voucher O. Khassanov, } \\
\text { 30.07.1959. №45 }\end{array}$} \\
\hline & & Fruits & fresh & stomach disorders & & \\
\hline \multicolumn{7}{|l|}{ Onagraceae } \\
\hline Epilobium angustifolium L. & Bargchoi & a.p. & decoction & $\begin{array}{l}\text { gastric ulcer, } \\
\text { inflammation of throat }\end{array}$ & & $\begin{array}{l}\text { specimen voucher U. Pratov, } \\
\text { 05.2001. №146 }\end{array}$ \\
\hline \multicolumn{7}{|l|}{ Orchidaceae } \\
\hline Dactylorhiza umbrosa Kar. \& Kir.) Nevski & Salib & tubers & decoction & cough, impotence & & $\begin{array}{l}\text { specimen voucher F. Karimov, } \\
\text { 07.06.2011. №5 }\end{array}$ \\
\hline
\end{tabular}




\begin{tabular}{|c|c|c|c|c|c|c|}
\hline Papaveraceae & & & & & & \\
\hline Fumaria vaillantii Loisel. & Shotara & a.p. & $\begin{array}{l}\text { decoction, } \\
\text { bath }\end{array}$ & $\begin{array}{l}\text { menstrual disorders as } \\
\text { bath for skin diseases }\end{array}$ & & $\begin{array}{l}\text { specimen voucher D. Jamalova, } \\
\text { 05.06.2006. }\end{array}$ \\
\hline Papaver pavoninum C.A. Mey. & Qizgaldak & flowers & extract & cough, common cold & & $\begin{array}{l}\text { specimen voucher R. Khalkuziev, } \\
\text { 24.05.1966. №108 }\end{array}$ \\
\hline Roemeria refracta DC. & Qizgaldok & flowers & extract & skin rash, fever & & $\begin{array}{l}\text { specimen voucher A. Ibragimov, } \\
\text { 05.06.2006. }\end{array}$ \\
\hline \multicolumn{7}{|l|}{ Plantaginaceae } \\
\hline Gratiola officinalis $\mathrm{L}$. & Safrouti & a.p. & decoction & diuretic, jaundice & $T$ & $\begin{array}{l}\text { specimen voucher V. Pasius, } \\
\text { 18.05.1932. №73. }\end{array}$ \\
\hline Plantago major L. & Bakayaprok & leaves & extract & $\begin{array}{l}\text { cough, external for } \\
\text { wounds }\end{array}$ & & $\begin{array}{l}\text { specimen voucher N. Beshko, } \\
\text { 18.07.1994. №62 }\end{array}$ \\
\hline Plantago lanceolata L. & Bargizub & leaves & extract & $\begin{array}{l}\text { asthma, cough, } \\
\text { external for wounds }\end{array}$ & & $\begin{array}{l}\text { specimen voucher U. Pratov, } \\
\text { 24.06.1996.№134 }\end{array}$ \\
\hline \multicolumn{7}{|l|}{ Polygonaceae } \\
\hline Polygonum aviculare L. & Qushtili & a.p. & decoction & blood coagulant & & $\begin{array}{l}\text { specimen voucher T. Sarybaev, } \\
\text { 08.06.1970. №43 }\end{array}$ \\
\hline P. coriarium Grig. & Toron & roots & extract & dysentery, diarrhea & $E$ & $\begin{array}{l}\text { specimen voucher A. Ibragimov, } \\
25.05 .2006 \text {. №192 }\end{array}$ \\
\hline Persicaria hydropiper (L.) Delarbre & Suv qalampir & a.p. & extract & $\begin{array}{l}\text { malaria, blood } \\
\text { coagulant }\end{array}$ & & $\begin{array}{l}\text { specimen voucher M. Nabiev, } \\
\text { 31.07.1954.№472 }\end{array}$ \\
\hline Rheum maximowiczii Losinsk. & Ravoch & roots & extract & diarrhea, malaria & $E, F$ & $\begin{array}{l}\text { specimen voucher A. Li, } \\
\text { 16.06.1960.№27 }\end{array}$ \\
\hline \multicolumn{7}{|l|}{ Punicaceae } \\
\hline Punica granatum $\mathrm{L}$. & Anor & bark, fruits & extract & $\begin{array}{l}\text { stomach diseases, } \\
\text { laxative }\end{array}$ & $\mathrm{F}$ & $\begin{array}{l}\text { specimen voucher S. Chevrenidi, } \\
\text { 18.08.1955. №503 }\end{array}$ \\
\hline \multicolumn{7}{|l|}{ Ranunculaceae } \\
\hline Adonis turkestanica (Korsh.) Adolf & Sank gul & a.p. & decoction & cardiac diseases & $\mathrm{E}, \mathrm{T}$ & $\begin{array}{l}\text { specimen voucher O. Turginov., } \\
\text { 11.05.2012.№314 }\end{array}$ \\
\hline Delphinium semibarbatum Bien. ex Boiss. & Isparak & a.p. & decoction & tonsillitis & $\mathrm{T}$ & $\begin{array}{l}\text { specimen voucher A. Batashov, } \\
\text { 05.05.2012.№211 }\end{array}$ \\
\hline \multicolumn{7}{|l|}{ Rhamnaceae } \\
\hline Rhamnus cathartica L. & Togjumrut & fruits & extract & $\begin{array}{l}\text { cancer, skin and } \\
\text { stomach diseases }\end{array}$ & $F$ & $\begin{array}{l}\text { specimen voucher T. Adylov, } \\
\text { 09.07.1972. №19 }\end{array}$ \\
\hline Ziziphus jujuba Mill. & Unabi & fruits & fresh, extract & $\begin{array}{l}\text { anemia, asthma, } \\
\text { kidney, liver diseases }\end{array}$ & $\mathrm{F}$ & $\begin{array}{l}\text { specimen voucher K. Toyjanov, } \\
\text { 19.09.1998. №265 }\end{array}$ \\
\hline
\end{tabular}




\begin{tabular}{|c|c|c|c|c|c|c|}
\hline Rosaceae & & & & & & \\
\hline Amygdalus communis $\mathrm{L}$. & Bodom & oil, seeds & fresh & asthma, cough, anemia & $\mathrm{F}$ & $\begin{array}{l}\text { specimen voucher U. Pratov, } \\
\text { 02.09.2005.№281 }\end{array}$ \\
\hline A. spinosissima Bunge & Bodomcha & seeds & fresh & anemia & & $\begin{array}{l}\text { specimen voucher K. Toyjanov, } \\
\text { 07.07.2006.№451 }\end{array}$ \\
\hline \multirow[t]{2}{*}{ Crataegus pontica Koch } & \multirow[t]{2}{*}{ Dulana } & flowers & ethanol & cardiac diseases, & \multirow[t]{2}{*}{$\mathrm{F}$} & \multirow{2}{*}{$\begin{array}{l}\text { specimen voucher N. Beshko, } \\
\text { 15.09.2012.№213 }\end{array}$} \\
\hline & & fruits & decoction & $\begin{array}{l}\text { hypertension, } \\
\text { sleeplessness }\end{array}$ & & \\
\hline \multirow[t]{2}{*}{ Crataegus turkestanica Pojark. } & \multirow[t]{2}{*}{ Dulana } & flowers & ethanol & cardiac diseases, & \multirow[t]{2}{*}{$E, F$} & \multirow{2}{*}{$\begin{array}{l}\text { specimen voucher A. Gazybaev, } \\
\text { 12.07.1991.№344 }\end{array}$} \\
\hline & & fruits & decoction & hypertension & & \\
\hline Potentilla anserina L. & Ak-dana & a.p. & extract & hemorrhage & & $\begin{array}{l}\text { specimen voucher U. Pratov, } \\
\text { 01.05.2002. №189 }\end{array}$ \\
\hline Rubus idaeus L. & Parmanchak & fruits & decoction & $\begin{array}{l}\text { common cold, cough, } \\
\text { fever }\end{array}$ & $\mathrm{F}$ & $\begin{array}{l}\text { specimen voucher A. Egamberdyev, } \\
\text { 11.06.2001. №211 }\end{array}$ \\
\hline Rosa canina L. & Itburun & fruits & decoction & $\begin{array}{l}\text { fever, stomach } \\
\text { disorders }\end{array}$ & & $\begin{array}{l}\text { specimen voucher K. Tojibaev, } \\
\text { 19.07.2009. №245 }\end{array}$ \\
\hline Rosa fedtschenkoana Regel & Namatak & fruits & extract & vitamin deficiency & & $\begin{array}{l}\text { specimen voucher A. Egamberdyev, } \\
\text { 13.06.2001.№95 }\end{array}$ \\
\hline Sorbus tianschanica Rupr. & Chetani & fruits & fresh, extract & vitamin deficiency & $\mathrm{F}$ & $\begin{array}{l}\text { specimen voucher U. Pratov, } \\
\text { 02.09.2005.№281 }\end{array}$ \\
\hline Spiraea hypericifolia L. & Tobulgi & $\begin{array}{l}\text { whole } \\
\text { plant }\end{array}$ & decoction & $\begin{array}{l}\text { stomach disorders, } \\
\text { vermifuge }\end{array}$ & & $\begin{array}{l}\text { specimen voucher K. Toyjanov, } \\
\text { 07.07.2006.№451 }\end{array}$ \\
\hline \multicolumn{7}{|l|}{ Rubiaceae } \\
\hline Rubia tinctorum Lapeyr. & Ruyan & rhizome & extract & $\begin{array}{l}\text { diuretic, dissolve kidney } \\
\text { stones }\end{array}$ & & $\begin{array}{l}\text { specimen voucher T. Adylov, } \\
\text { 05.07.1957. №582. }\end{array}$ \\
\hline \multicolumn{7}{|l|}{ Rutaceae } \\
\hline Dictamnus albus L. & Togturbid & $\begin{array}{l}\text { whole } \\
\text { plant }\end{array}$ & extract & $\begin{array}{l}\text { epilepsy, jaundice, } \\
\text { snake bite }\end{array}$ & & $\begin{array}{l}\text { specimen voucher I. Maltsev, } \\
\text { 10.09.1998. №127 }\end{array}$ \\
\hline \multicolumn{7}{|l|}{ Saxifragaceae } \\
\hline Bergenia ugamica V.N. Pavlov & Badan & roots & decoction & $\begin{array}{l}\text { menstrual disorders, } \\
\text { immuno-stimulant }\end{array}$ & $\mathrm{E}$ & $\begin{array}{l}\text { specimen voucher I. Maltsev, } \\
\text { 10.09.1998. №127 }\end{array}$ \\
\hline \multicolumn{7}{|l|}{ Scrophulariaceae } \\
\hline Verbascum songaricum Schrenk & Sigir kuyruk & flowers & decoction & $\begin{array}{l}\text { cough, external for } \\
\text { wounds }\end{array}$ & & $\begin{array}{l}\text { specimen voucher L. Nazarenko, } \\
\text { 27.06.1970. №185 }\end{array}$ \\
\hline
\end{tabular}




\begin{tabular}{|c|c|c|c|c|c|c|}
\hline Solanaceae & & & & & & \\
\hline Datura stramonium L. & Bangidevona & leaves & extract & $\begin{array}{l}\text { external use only for } \\
\text { rheumatism }\end{array}$ & $\mathrm{T}$ & $\begin{array}{l}\text { specimen voucher H. Esanov, } \\
\text { 12.05.2006. №82. }\end{array}$ \\
\hline Hyoscyamus niger L. & Mingdevona & leaves & extract & $\begin{array}{l}\text { external use only for } \\
\text { rheumatism }\end{array}$ & $\mathrm{T}$ & $\begin{array}{l}\text { specimen voucher R. Khalkuziev, } \\
\text { 02.06.1984. №99 }\end{array}$ \\
\hline \multicolumn{7}{|l|}{ Urticaceae } \\
\hline Urtica dioica L. & Gazanda ut & leaves & extract & $\begin{array}{l}\text { blood coagulant, } \\
\text { vitamin deficiency }\end{array}$ & & $\begin{array}{l}\text { specimen voucher H. Esanov, } \\
\text { 17.07.2009. №405 }\end{array}$ \\
\hline \multicolumn{7}{|c|}{ Zygophyllaceae } \\
\hline \multirow[t]{2}{*}{ Peganum harmala L. } & \multirow[t]{2}{*}{ Issiriq } & a.p. & smoke, bath, & influenza, malaria, & \multirow[t]{2}{*}{$\mathrm{T}$} & \multirow{2}{*}{$\begin{array}{l}\text { specimen voucher L. Botirova, } \\
\text { 06.2010. №512 }\end{array}$} \\
\hline & & & decoction & as bath for itching & & \\
\hline
\end{tabular}

Legend: E - Endemic plants, F - Food, T - Toxic 
Taxonomically the 117 species belonged to 45 families and 94 genera. The families which were more frequently used are shown in Table 2. Medicinal plants from Amaryllidaceae, Asteraceae, Lamiaceae and Rosaceae are prevailing in usage in Central Asia (Keusgen et al. 2006.; Ryabushkina et al. 2008; Sakhobiddinov 1948; Sharopov et al. 2015). Local people interviewed in Uzbekistan (Table 3) were using traditional remedies mainly for the treatment of respiratory, gastrointestinal and liver diseases. Medicinal plants used to treat cancer still are a very recent emergence.

Table 2. The most using plant families of medicinal plants

\begin{tabular}{llll}
\hline Family & Genera & Species & $\%$ \\
\hline \hline Amaryllidaceae & 2 & 4 & $3,42 \%$ \\
Apiaceae & 5 & 5 & $4,27 \%$ \\
Asteraceae & 14 & 18 & $15,38 \%$ \\
Fabaceae & 6 & 8 & $6,84 \%$ \\
Juglandaceae & 6 & 4 & $3,42 \%$ \\
Lamiaceae & 10 & 10 & $8,55 \%$ \\
Polygonaceae & 2 & 4 & $3,42 \%$ \\
Rosaceae & 7 & 10 & $8,55 \%$ \\
\hline Total & 52 & 63 & $53,85 \%$
\end{tabular}

Table 3. Rank-ordered list of folk remedies according to group of ailments employed for the treatment

\begin{tabular}{lccc}
\hline Diseases & Genera & Species & $\%$ \\
\hline \hline Cardiovascular & 11 & 12 & $7,36 \%$ \\
Oro-dental & 3 & 3 & $1,84 \%$ \\
Skin & 12 & 12 & $7,36 \%$ \\
Endocrine system & 6 & 6 & $3,68 \%$ \\
Gastrointestinal & 21 & 23 & $14,11 \%$ \\
Genital-urinary & 12 & 12 & $7,36 \%$ \\
Infectious & 6 & 6 & $3,68 \%$ \\
Liver & 17 & 19 & $11,66 \%$ \\
Nervous system & 9 & 9 & $5,52 \%$ \\
Tumours & 2 & 2 & $1,23 \%$ \\
Respiratory & 22 & 25 & $15,34 \%$ \\
Other & 30 & 34 & $20,86 \%$ \\
\hline Total & 151 & 163 & $100,00 \%$
\end{tabular}

For the most used plants in folk medicine and exportoriented species (Ungernia victoris, Ferula foetida, Helichrysum maracandicum, Capparis spinosa, Ephedra equisetina, Glycyrrhiza glabra), we assessed the current state of natural populations in the regions of the Republic of Uzbekistan. As a result of the study, we identified opportunities for the annual exploitation of wild resources, compiled GIS maps and proposed measures for sustainable use, which in the future will optimize the production of raw materials and preserve existing plant populations.

The obtained data serve as the basis for further ethnobotanical research to identify original data on the use of medicinal plants in folk medicine in treatment. Established reliable data through analysis will be proposed for further in-depth study by phytochemists, pharmacologists and toxicologists, which in the future will replenish the arsenal of medicines of scientific medicine.

\section{Discussion}

Around 100 different nationalities live in Uzbekistan, and ethnic differences explain to some extent the wide spectrum of medicinal plant uses found. Traditional medicine has been an invaluable segment of healthcare in the past and needs to be documented to prevent the disappearance of knowledge. However, our study indicates that medicinal plant knowledge is still widespread in Uzbekistan. After mostly focusing on chemically synthesized medicines in the $20^{\text {th }}$ century, the pharmaceutical industry is slowly shifting towards herbal preparations, in particular in the form of herbal supplements, and the interest in natural remedies and alternative healing methods is increasing throughout the western world. In addition, despite great advances in the chemical industry, some plant substances, e.g. the compounds found in Valerian root (Valeriana officinalis L.), or their natural combinations are unique, and yet non-replicable by synthetic chemistry. 
Like in many regions worldwide, the population of Uzbekistan is facing the spread of cardiovascular and gastrointestinal diseases. For treatment of these diseases, people are frequently using the aerial parts of the Hypericum scabrum (Matsuhisa et al. 2002; Tanaka et al. 2004), Leonurus turkestanicus and Adonis turkestanica (Figure 3). Using samples collected during our investigation, it was found that Bergenia ugamica (Figure 4) contains substances with immuno-stimulating activity, confirming the oral information of participants, who indicated that the root of Badan (B. ugamica) make the human body stronger to defend against diseases.

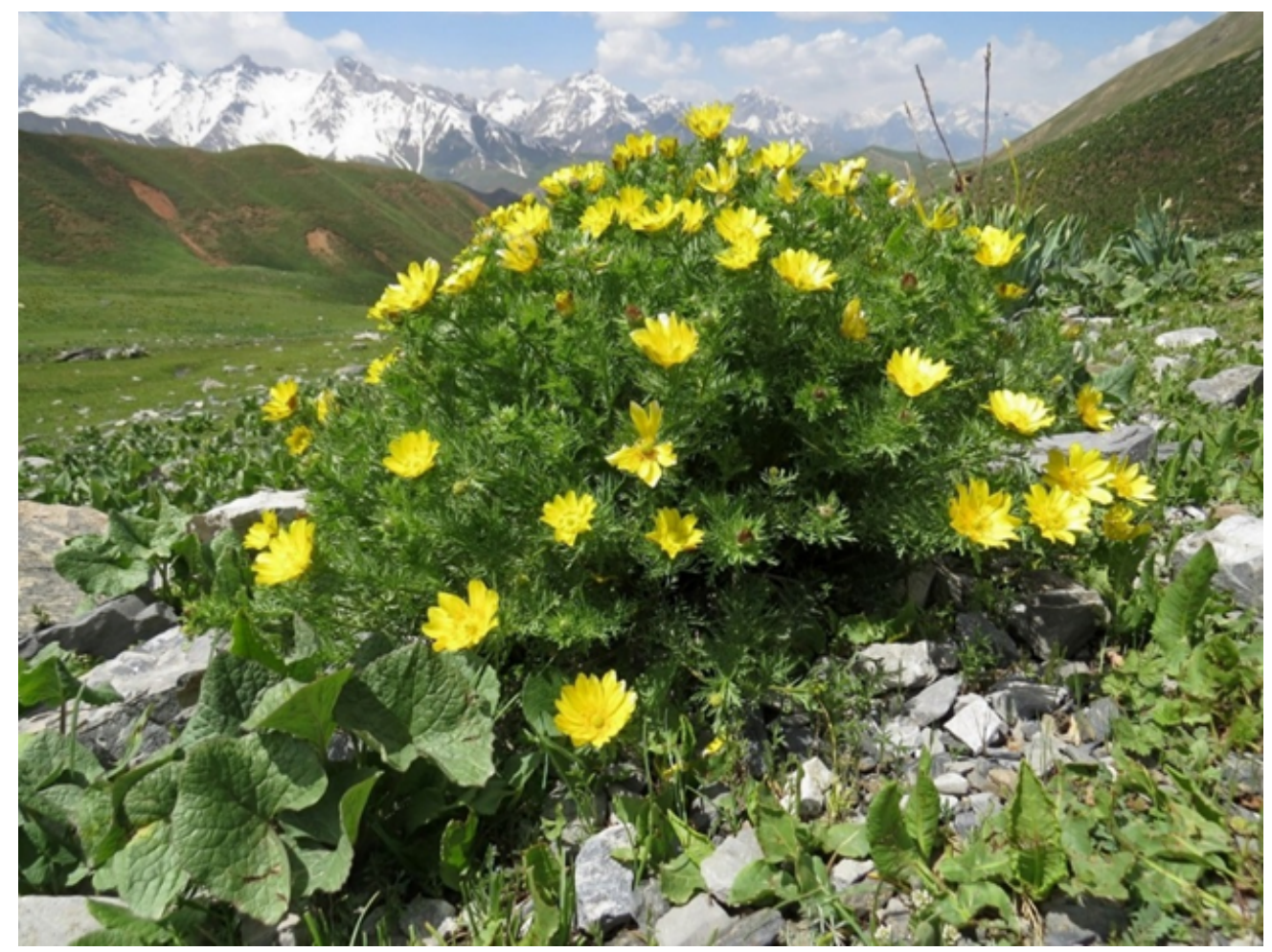

Figure 3. Flowers of Adonis turkestanica (Korsh.) Adolf.

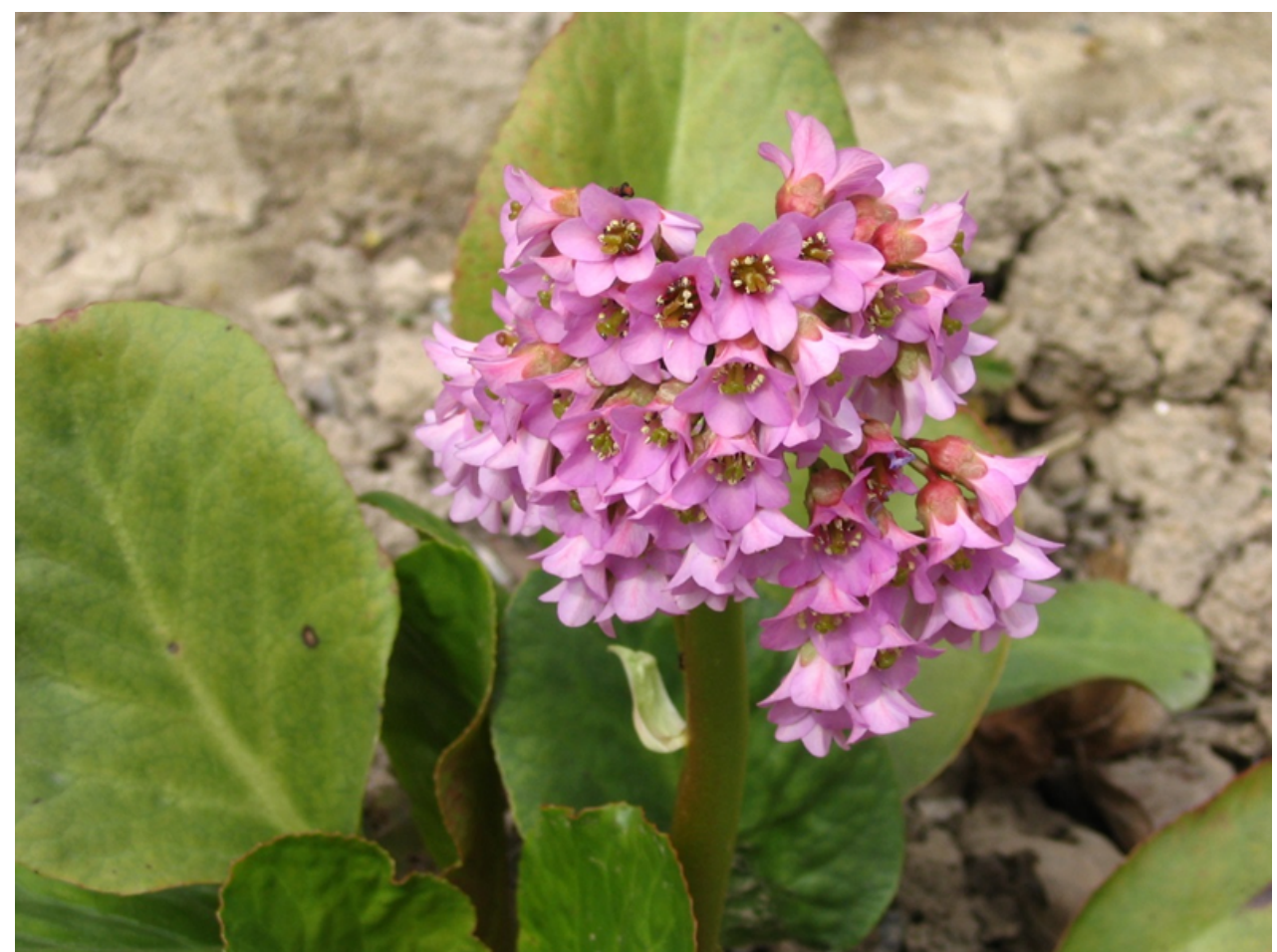

Figure 4. Flowers of Bergenia ugamica V.N. Pavlov 
Several endemic species are still widely used as astringents and coagulants (e.g. Bozulbang Lagochilus inebrians). All parts of $L$. inebrians contain the diterpene-alcohol lagochilin, alkaloids, essential oils, tannins, resins, $\mathrm{Ca}, \mathrm{Mg}$ and 20 different microelements ( $\mathrm{Co}, \mathrm{Ti}, \mathrm{Au}$ ), vitamins $\mathrm{C}$ and $\mathrm{K}$, and organic acids (Kholmatov et al. 1984). Drugs prepared from Bozulbang are widely used in official public healthcare as blood coagulant, sedative for nervous diseases and for treatment various skin diseases as eczema, itching, etc.

Another endemic species, naturally growing in Pamir-Alay (Hissar ridge), is Omonkora - Ungernia victoris (Figure 5). Ethnobotanical information about this plant indicates that traditionally the population is still using baked bulbs and fresh leaves for the disinfection of wounds. Local people are also still making glue from bulbs. All parts of $U$. victoris contain alkaloids (leaves-0,33\%-1\%; bulbs $0,8 \%$ $0,9 \%$, especially galantamin $0,7 \%-1 \%$, licorin $0,073 \%$, gordenin $0.039 \%$, tatecin $0,1 \%$, pankratin $0,15 \%$, narvedin $0,0054 \%$ ). The maximum concentration of alkaloids in $U$. victoris can be found in early spring. This plant also contains cumarin $(0,09 \%)$, essential oils $0,12 \%$, resins $6 \%$, pectin $4,9 \%$, mucus $7 \%$, sugars $6,1 \%$ and organic acids $8,91 \%$ (Khojimatov et al. 2009). The leaves and bulbs of the plant are now used as an industrial source of the alkaloid galantamin, which is widely used in allopathic medicine for treatment of poliomyelitis and polyneuritis.

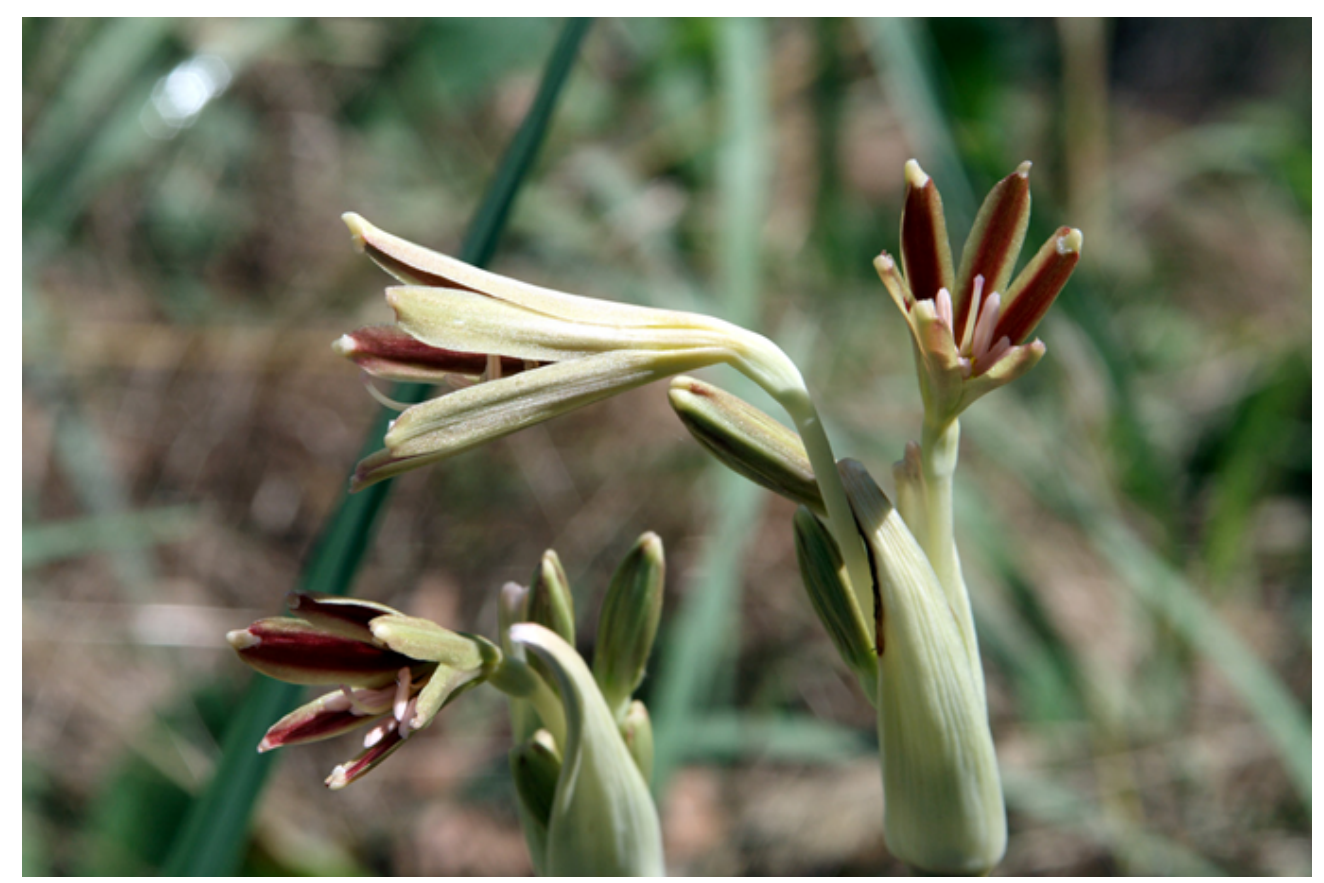

Figure 5. Flowers of Ungernia victoris Vved.

Another endemic species Alkoruti - Mediasia macrophilla (Figure 6) is usually added to milk or dairy products to keep them fresh. It is also used as medicine by local people in Uzbekistan. The aerial parts have been used traditionally as a perfume, an appetite enhancer, as a natural preservative, and for treatment of rheumatism, nephritis, and eczema (Kurimoto et al. 2010).

Only recently Codonopsis bactriana (Figure 7) was described. This species is closely related to Tien Shan - Dzhungarian C. clematidea (Schrenk.) C.B. Clarke, which is found commonly in the Western Pamir-Alay (Uzbekistan and Tajikistan) and used by tabibs for the treatment of different kinds of liver diseases, such as hepatitis, jaundice and the first stage of liver cirrhosis. Such traditional knowledge has already found its way into the market economy.
Ethnobotanical studies provided the base for the development of an original and effective "Species cholagogae Chodjimatovii," which has been in use since 2002. This drug has shown high efficacy in the treatment of various forms of hepatitis, the initial stages of cirrhosis and diseases of the biliary tract. It was patented on 26.03.2006, No. IAP 02046, registration certificate dated September 30, 2017 No. 02/548/17 (Figure 8).

Another endemic species Buzbosh Dracocephalum komarovii (Figure 9) is a perennial spiny semi-shrub growing in the alpine zone at 3000$3600 \mathrm{~m}$ in the West Tien Shan mountain system. Local people are using aerial parts in a tea to cure various disorders such as inflammatory diseases and hypertony (Khojimatov et al. 1995; Uchiyama et al. 2003). 


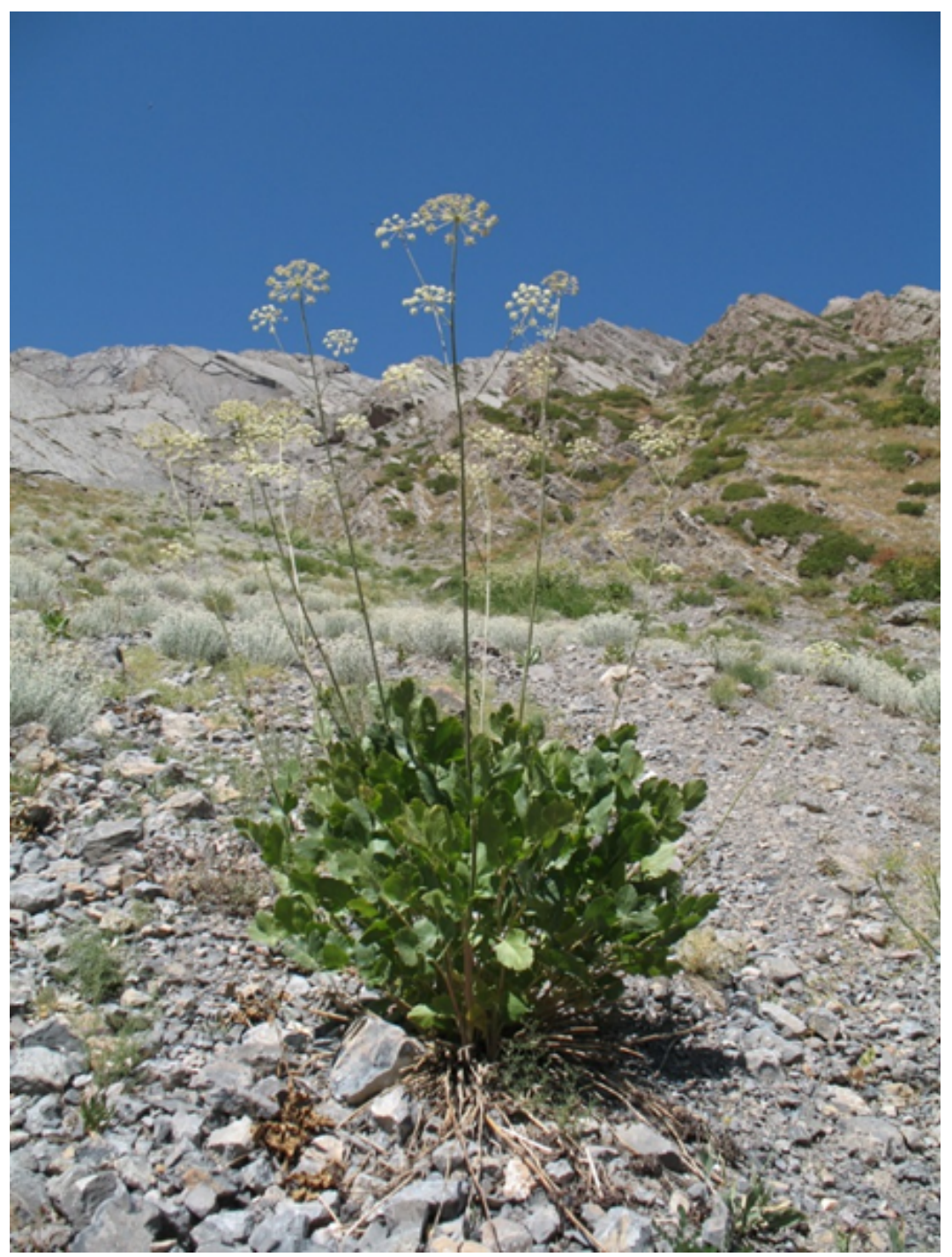

Figure 6. Mediasia macrophylla (Regel \& Schmalh.) Pimenov

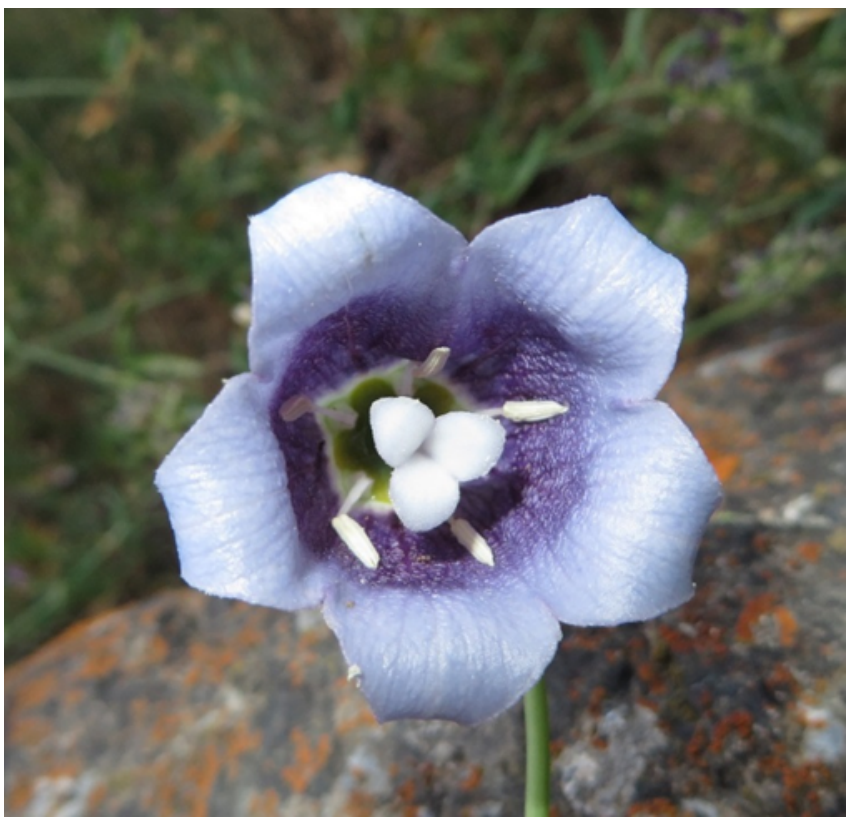

Figure 7. Flower of Codonopsis clematidea (Schrenk) C.B. Clarke 


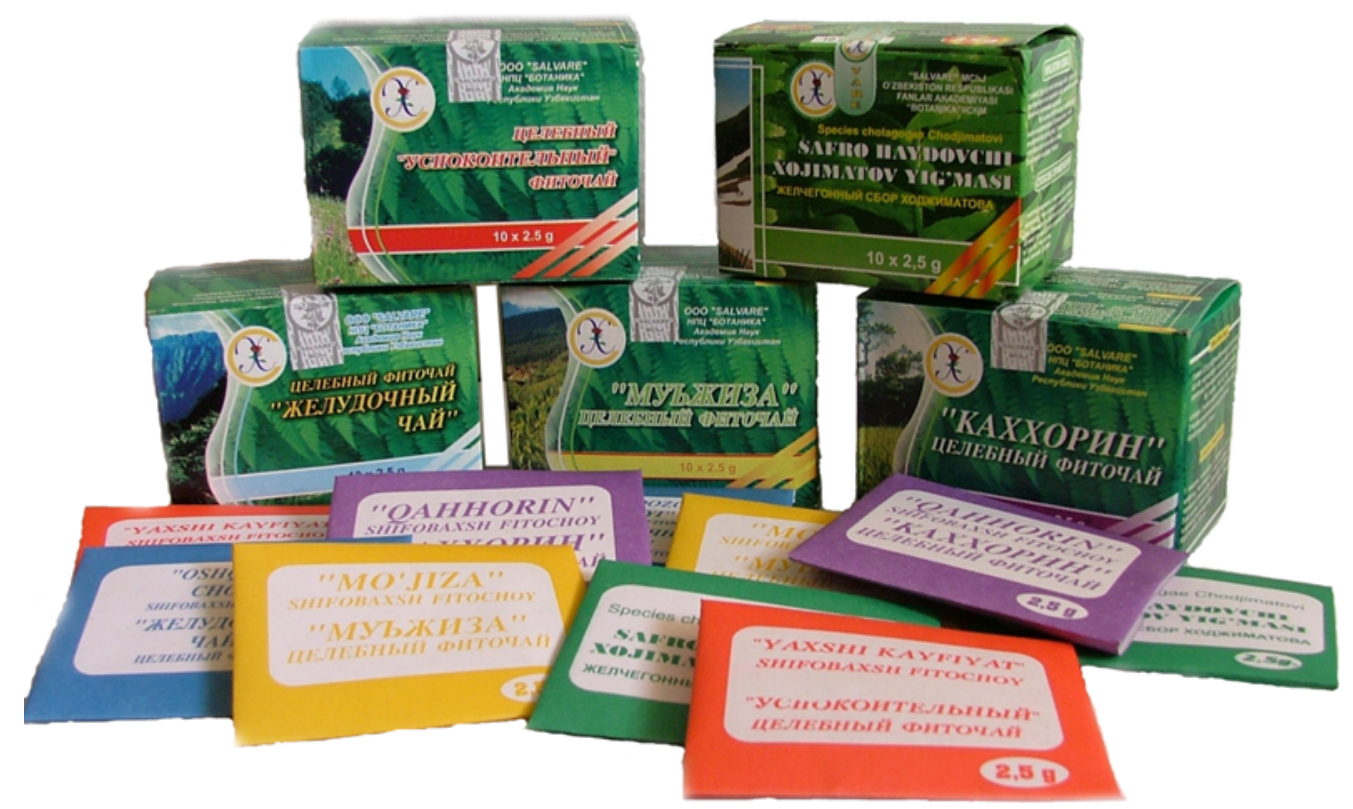

Figure 8. Species cholagogae Chodjimatovii

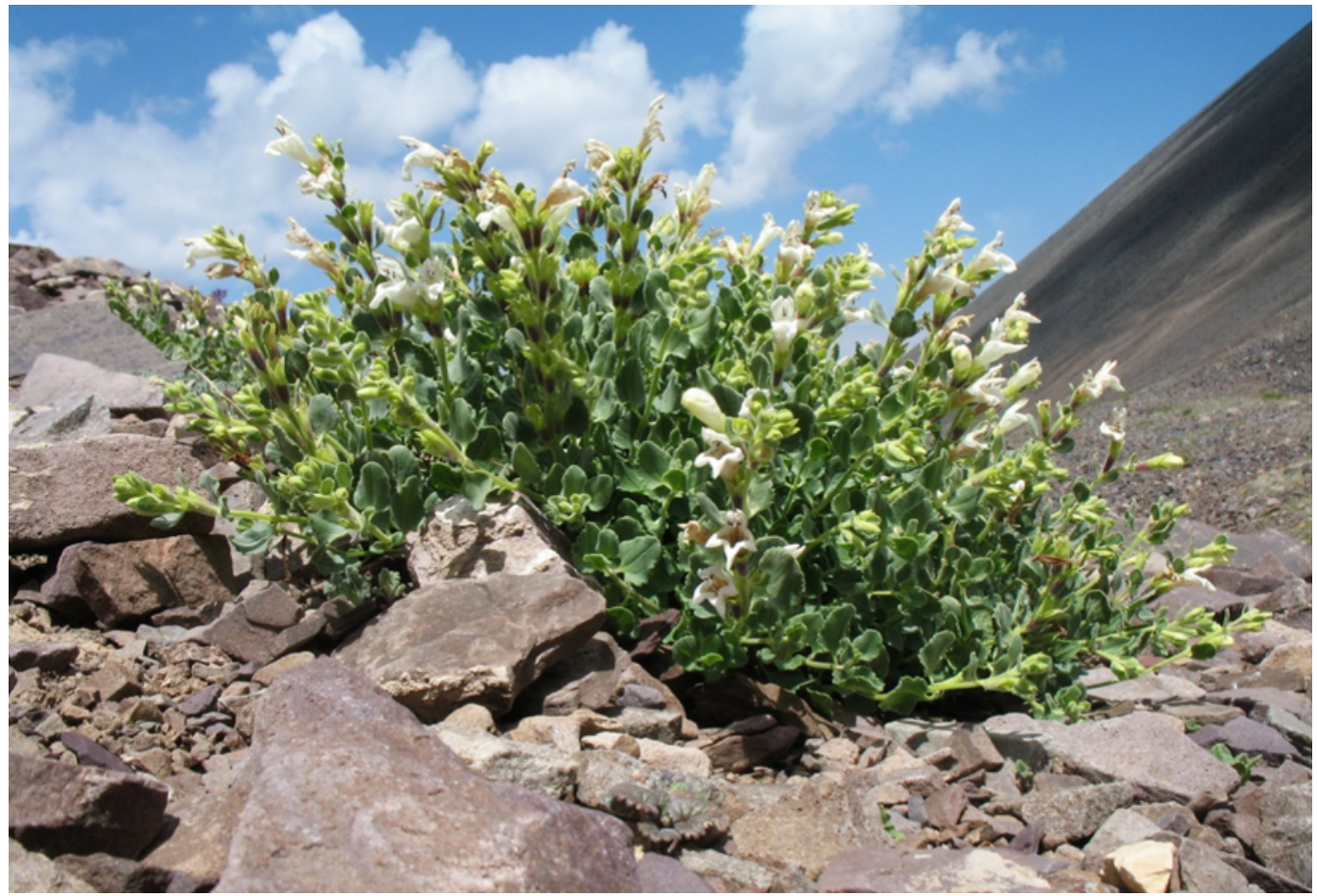

Figure 9. Dracocephalum komarovii Lipsky

In order to standardize the available ethnobotanical information, the Institute of Botany (Tashkent) has developed a database containing detailed information for each plant, e.g. scientific names (accepted name and, when necessary, main synonyms), common name used in literature, and any other names commonly in use, geographical distribution, morphological description, healing properties, traditional medicinal usage and known adverse effects, information on scientifically proven and medicinally approved data, and information received from sources related to traditional medicine, including associated remedies and recipes.

\section{Conclusions}

Our results show that despite the achievements of modern allopathic medicine, the population of Central Asia continues to actively use wild medicinal plants for health improvement. This refutes our hypothesis that knowledge is very widely lost. The 
decisive factors for the choice and acceptance of herbal drugs are primarily the centuries-old experiences that forms the basis of traditional medicine. This is followed by a wide range of plant effects, that, in most cases, low toxicity of plant preparations, their low cost, and easy availability. All these factors contribute to the increasing popularization of treatments with medicinal plants, both in pure form and as part of multicomponent preparations. We consider it very important to continue ethnobotanical research, covering more and more territories and involving more participants who possess unique knowledge in the field, which may in the future help to develop new effective drugs based on centuries-old experience of folk medicine.

\section{Declarations}

List of abbreviations: TASH - Herbarium of the Institute of Botany Academy of Sciences, Uzbekistan.

Ethnics approval and consent to participate: All participants involved in the interview process gave their prior informed oral consent.

Funding: Not applicable.

Availability of data and materials: The data was not deposited in public repositories but is available from the corresponding author upon request.

Authors' contributions: OK Khojimatov collected and analyzed the data, drafted and developed the manuscript. DT Khamraeva searched literature, cooperated in data collection and adjusted the manuscript to the journal submission guidelines. AN Khujanov analyzed the data and structured the tables. RW Bussmann critically revised the manuscript. All authors contributed in the research proposing the problem, data collection, and approved the final manuscript.

Consent for publication: Not applicable.

Competing interests: The authors declare that they have no competing interests.

\section{Acknowledgements}

This work is part of the research capacity building "Compilation of an interactive atlas of promising medicinal plants of Uzbekistan (resources, monitoring and methods of protection of the most vulnerable species)", which receives financial support from Ministry of innovative Development of the Republic of Uzbekistan, Grant number П3 20170920219.

\section{Literature cited}

Angiosperm Phylogeny Group. 2009. An update of the Angiosperm Phylogeny Group classification for the orders and families of flowering plants: APG III. Botanical Journal of the Linnean Society 161(1):105121.
Astulla A, Zaima K, Matsuno Y, Hirasawa Y, Ekasari W, Widyawaruyanti A, Zaini NCh, Morita H. 2008. Alkaloids from the seeds of Peganum harmala showing antiplasmodial and vasorelaxant activities. Journal of Natural Medicines 62(4):470-472.

Ibn Sina AA. 1982. Canon of Medicinal Science. Tashkent, Fan. Vol. 2.

Keusgen M, Fritsch RM, Hisoriev $\mathrm{H}$, Kurbonova PA, Khassanov FO. 2006. Wild Allium species (Alliaceae) used in folk medicine of Tajikistan and Uzbekistan. Journal of Ethnobiology and Ethnomedicine 2(1):18. doi:10.1186/1746-4269-218

Khassanov F. 2015. Conspectus Florae Asiae Mediae. Vol.11, Tashkent, Fan.

Khojimatov K, luldoshev K, Shogulomov U, Khojimatov O. 1993. Shifobahsh giyohlar qand hastaliga malham (in Uzbek). Tashkent, Semashko Institution Press.

Khojimatov K, luldoshev K, Shogulomov U, Khojimatov O. 1995. Shifobahsh giyohlar-dardlarga malham (in Uzbek). Tashkent, Uzbekiston.

Khojimatov K, Kobetc L. 1988. Napitki iz trav (in Russian). Tashkent, Mekhnat.

Khojimatov K, Olloyorov M. 1988. Uzbekistonning shifobahsh usimliklari va ularni muhofaza kilish (in Uzbek). Tashkent, Fan.

Khojimatov O. 2008. Lekarstvennye rasteniya YugoZapadnogo Tyan-Shanya ( $v$ predelax Respubliki Uzbekistan). (in Russian). Diss. of DSc. Tashkent, $322 \mathrm{p}$.

Khojimatov O, Khojimatov K, Sobirov U. 2009. Sbornik pravil poljzovania objektami lekarstvennyh, pishevyh i tehnicheskih rastenuj (in Russian). Tashkent, Yangy asr avlody.

Khojimatov OK, Abdiniyazova GJ, Pak VV. 2015. Some wild growing plants in traditional foods of Uzbekistan. Journal of Ethnic Foods 2:25-28. doi: 10.1016/j.jef.2015.02.005

Kholmatov K, Kharlamov I, Alimbaeva P, Karriev M, Khaitov IKh. 1984. Osnovnie lekarstvennie rastenia Srednei Azii (in Russian). Tashkent.

Kurimoto S, Okasaka, M, Kashiwada Y, Kodzhimatov O, Takaishi Y. 2010. C14polyacetylenic glucoside with an alpha-pyrone moiety and four C10-polyacetylenic glucosides from Mediasia macrophylla. Phytochemistry 71(5-6):688692.

Kurimoto S, Okasaka M, Kashiwada Y, Kodzhimatov OK, Takaishi Y. 2011. Four new glucosides from the aerial parts of Mediasia macrophylla. Journal Nat. Medicine 65(1):180-185. doi: 10.1016/j.phytochem.2009.12.007

Matsuhisa M, Shikishima Y, Takaishi Y, Honda G, Ito M, Takeda Y, Shibata H, Higuti T, Khodzhimatov OK, 
Ashurmetov O. 2002. Benzoyl phloroglicinol derivatives from Hypericum scabrum. Journal of Natural Products 65(3):290-294. PMID:11908967. doi:10.1021/np010310

Moloudizargari M, Mikaili P, Aghajanshakeri Sh, Asghari MH, Shayegh J. 2013. Pharmacological and Therapeutic effects of Peganum harmala and its main alkaloids. Pharmacognosy Reviews 7(14):199212. doi:10.4103/0973-7847.120524

Pak VV, Khojimatov OK, Abdiniyazova GJ, Magay EB. 2019. Composition of camel milk and evaluation of food supply for camels in Uzbekistan. Journal of Ethnic Foods 6(20). doi: 10.1186/s42779-019-00315

Ryabushkina N, Gemedjieva N, Kobaisy M, Cantrell Ch. 2008. Brief review of Kazakhstan flora and use its wild species. The Asian and Australasian Journal of Plant Science and Biotechnology 2(2):64-71.

Sakhobiddinov SS. 1948. Dikorastushie lekarstvennie rasteniya Sredney Azii. Gosizdat UzSSR, Tashkent.

Sharopov F, Zhang H, Wink M, Setzer WN. 2015. Aromatic medicinal plants from Tajikistan (Central Asia).. Medicines 2:28-46. doi:10.3390/medicines2010028.

Tanaka N, Takaishi Y, Shikishima Y, Nakanishi Y, Bastow K, Lee K-H, Honda G, Ito M, Takeda $Y$, Khodzhimatov OK, Ashurmetov O. 2004. Prenylated benzophenones and xanthones from Hypericum scabrum. Journal of Natural Products 67(11):18701875. doi: $10.1021 / \mathrm{np} 040024$

Uchiyama N, Kiuchi F, Ito M, Honda G, Takeda Y, Khodzhimatov OK, Ashurmetov O.A. 2003. New icetexane and 20-norabietane diterpenes with trypanocidal activity from Dracocephalum komarovii. Journal of Natural Products 66(1):128-131. doi:10.1016/j.tet.2006.02.067

Uchiyama N, Kiuchi F, Ito M, Honda G, Takeda Y, Khodzhimatov OK, Ashurmetov OA. 2006. Trypanocidal constituents of Dracocephalum komarovii. Tetrahedron 62(18):4355-4359. doi:10.1016/j.tet.2006.02.067

Vavilov, N. I. (1992). Origin and geography of cultivated plants. Cambridge University Press,

Cambridge, UK. 